\title{
25 years of HIV
}

\section{Reflecting on how far we have come scientifically since isolating HIV in 1983, Anthony S. Fauci urges a renewed commitment to the far greater challenges ahead, especially that of vaccine development.}

\begin{abstract}
1 he HIV/AIDS catastrophe has been one of the defining features of the past quarter of a century. Although it is short-lived in the scheme of public-health crises, the pandemic ranks among the most devastating microbial scourges in human history, one whose full impact has yet to be realized.

Sixty million people have been infected with the human immunodeficiency virus (HIV); nearly half have died, and the toll on families, communities and even entire nations has been profound. Meanwhile, the biomedical research effort directed at HIV/AIDS has resulted in some breathtaking successes. Unlike many other diseases that affect mostly the poor, marginalized and disenfranchised, HIV/AIDS captured the attention of world leaders, the medical, public-health and activist communities, funding agencies, phi-
\end{abstract} lanthropists and many celebrities. This resulted in an unprecedented scientific and public-health response to the disease, and in welcome attention to some of the many other problems endemic in those populations most severely afflicted with HIV/AIDS, such as malaria, tuberculosis and gender inequality.

Much remains to be accomplished in the global fight against HIV. There are many more scientific and medical hurdles to be cleared and numerous logistical and operational obstacles to making therapies and other interventions available to poor countries, where per capita income is sometimes only a few hundred dollars a

"HIV/AIDS is
predominantly a disease
of the developing
world, where access to
scientific advances and
therapies is difficult."

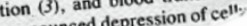
pronounced depre in and the $m$

tors from different disciplines began working on this new disease. Within months of the recognition of the first cases in the summer of 1981, I shifted the direction of my laboratory from the study of inflammatory diseases to this curious new syndrome. My research has been closely intertwined with HIV and AIDS ever since. For nearly two years, the cause of AIDS remained elusive; the scientific community was largely baffled, lacking good leads for developing therapies or even a diagnostic test. Those of us caring for patients with AIDS had few tools at our disposal. The only treatments we could provide were largely palliative (AIDS) has recently been recog- teell leukemia. Aralysis of the prolated in nized in several countries (f). This virus showed them from ease has been reported maic partsers, and but clearly dist isolate exual males with multiple parts and previous HTLV isolat sexual milogical studies suggest hor (2) as recent studin epidemiogition by sextal roules (2) as cail transmissingavenous drug administrawell as by ind blood transfusion (A)

of the AIDS pandemic. Only in retrospect can we identify its different stages. The first began in June 1981, when physicians in New York and California reported unusual clusters of rare diseases in previously healthy gay men, notably Pneumocystis carinii pneumonia and a form of cancer called Kaposi's sarcoma. When we in the medical profession read those initial cases 27 years ago - and treated some of the early AIDS patients - our prevailing emotion was bewilderment.

I had seen other 'mystery' diseases in my career, such as the legionnaires' disease outbreak of 1976. But AIDS was from the beginning year and health-care spending a tiny fraction of that. Reflecting on the era of HIV/AIDS, we must learn from our mis-steps, build on our successes in treatment and prevention, and renew our commitment to developing the truly transforming tools that will one day put this scourge behind us.

\section{Baffling beginnings}

People living through historic events often fail to recognize the significance of what they experience. Such was the case for me, and many of my colleagues, in the first months much more insidious and enigmatic. As cases began to appear among distinctly different social groups in 1981-82, and as we began to understand better the profound and complex immunodeficiency of our patients, it became clear that we were witnessing the unfolding of something truly novel and frightening. The severity of AIDS and the signs that it apparently could be spread by a ubiquitous human activity - sex - suggested that we were in for a difficult time.

The scientific community marshalled its resources and talent to fight AIDS; investiga- and the lifespan of most of our patients was measured in months. Those years were the darkest of my professional career.

\section{Glimmer of hope}

Twenty-five years ago this month came a glimmer of hope. In 1983, Luc Montagnier's research team in Paris published in Science the first paper (pictured) providing evidence linking a retrovirus to AIDS. The following year, further data from Robert Gallo's group in the United States provided convincing evidence that this retrovirus (later named HIV) was the cause of AIDS. That these two outstanding scientists became embroiled in a controversy largely played out in the media - about who discovered HIV was an unfortunate distraction. As they would later write in The New England Journal of Medicine: "Many lessons can be drawn from this early intense period, and most suggest that science requires greater modesty." A quarter of a century on, the importance of collaboration, collegiality and, yes, modesty, are ever more apparent, as it becomes clear that no single research group or discipline will solve the puzzles of HIV/AIDS.

After the discovery of HIV, research moved at a breathtaking pace. A blood test to diagnose patients and to screen the blood supply quickly followed, as did enormous progress in understanding the genetics and structure of HIV and its disease-causing mechanisms. The rapid clinical testing and licensing in 1987 of the first effective drug against HIV, zidovudine (AZT), caused great excitement. In retrospect this was unfounded, as the molecular characteristics 
of HIV, notably its propensity to replicate and mutate rapidly, made any single drug unlikely to hold the virus in check. Previous experience with antimicrobials for other diseases and the inevitable emergence of drug-resistant pathogens should have made us more cautious about the prospects for AZT monotherapy.

HIV quickly developed resistance to AZT and the benefits of the drug rapidly waned. Initial optimism about therapy gave way to sobering reality as the AIDS pandemic continued to grow in the United States and elsewhere. Clinicians remained hobbled by a lack of effective anti-HIV drug regimes, and many more patients were lost to AIDS.

Gradually, the fruits of cutting-edge drug development began to appear. In late 1995, the first of a new class of antiretroviral drugs - protease inhibitors - reached the market. Other new drugs that attacked the virus in different ways followed, and we soon had a greater number of effective drugs for HIV than for all other viral diseases combined. The new therapies used in combination with older medicines rapidly improved the prognosis for vast numbers of HIV-infected patients. The AIDS death rate in the United States fell by more than two-thirds within two years of the licensing of the first protease inhibitor. Despite certain limitations of the new treatments, notably toxicity and drug resistance, they launched a new era of optimism.

But HIV/AIDS is predominantly a disease of the developing world, where access to scientific advances and therapies is difficult. Fewer than one-third of the people who need antiretroviral therapy are currently receiving it, despite heroic efforts on the part of individuals and organizations, and some truly transforming and innovative programmes such as the Global Fund to Fight AIDS, Tuberculosis, and Malaria and the US President's Emergency Plan for AIDS Relief. Furthermore, it is clear that treatment alone will never end the AIDS pandemic. New infections far outstrip our ability to treat everyone infected with the virus: around three people are newly infected for every person put on therapy - and current HIV therapy is a lifelong commitment.

\section{More mountains}

To improve these formidable odds, we have two main options. The first is to cure patients, that is, to purge every vestige of virus from their bodies so that a course of treatment could be measured in weeks or months rather than

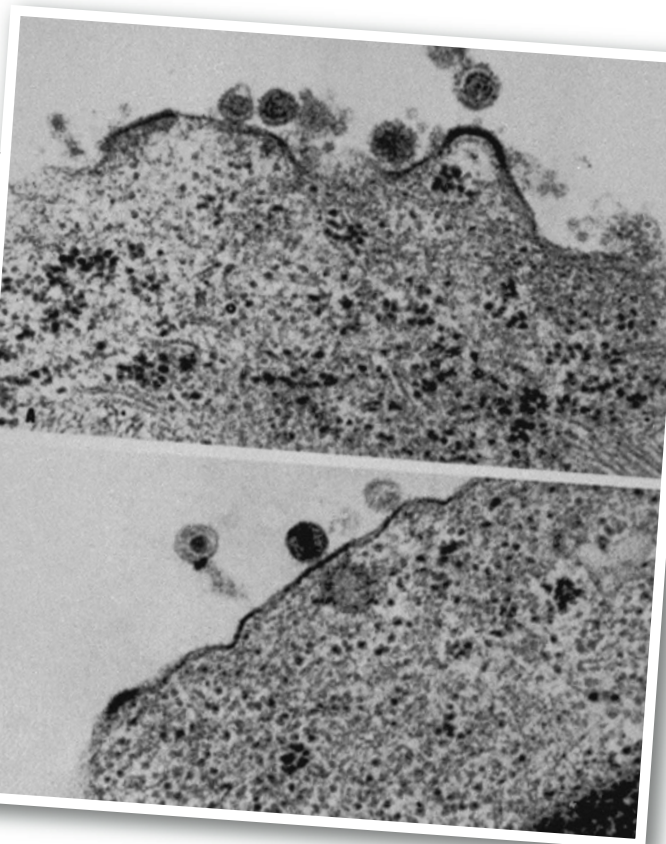

Agent identified: early images captured by Robert Gallo (top) and Luc Montagnier of the retrovirus later named HIV.

a lifetime. Sadly, because of the ability of HIV to hide within cells from both drugs and the immune system, such a treatment regime has proved elusive, although important work in this area is being pursued.

This leaves us with the second option: preventing HIV infection in the first place. We have a proven array of HIV-prevention and harm-reduction strategies: behavioural modification; condom distribution; antiretroviral drug regimes to prevent HIV transmission from mother to baby; and the provision of clean needles and syringes to drug users. We must now do better at delivering prevention: less than $20 \%$ of those at risk of HIV infection are currently receiving such help.

Encouragingly, new means of preventing HIV are emerging. Large randomized and

"The major goal of HIV/AIDS
research eludes us: the
development of a safe and
effective HIV vaccine."
controlled clinical trials in Africa suggest that adult male circumcision, if properly and hygienically performed and accompanied by appropriate counselling and post-surgical care, can help prevent men becoming infected with HIV by heterosexual intercourse. Ongoing work to develop microbicidal gels or creams to be applied before sex offers the hope of empowering women to protect themselves from HIV infection when the use of condoms or the refusal of sexual intercourse is not feasible.

Yet the major goal of HIV/AIDS research eludes us: the development of a safe and effective HIV vaccine, our best hope for ultimately ending the pandemic. The search for a vaccine has been made extremely difficult by the nature of the virus, particularly its ability to integrate into the genome of host cells, to mutate readily and to conceal that part of its outer coat that would induce protective antibodies.

It is now clear that we were naive to think there would be a straight path from the discovery and characterization of HIV to the development of a vaccine. HIV has proved very different from those viruses for which we have developed effective immunizations. We must solve the mystery of how to prompt the human body to produce a protective immune response that is even better than the one elicited by natural infection. This will require a commitment to fundamental research to address the many questions that remain about HIV and its interactions with its human host. HIV/AIDS science, particularly that involving a vaccine, is in some ways still in its infancy. We must move forward by fostering creative thinking over many different disciplines.

In this regard, the pursuit of new research avenues by established scientists and especially by younger investigators is critical. In addition to the disciplines classically associated with HIV research - virology and immunology - we must encourage more 'cross-fertilization' with other fields such as genetics, structural biology, systems biology and peptide chemistry as we strive to generate the knowledge needed to develop an HIV vaccine.

Delivering HIV interventions for the people it most affects requires political will, a long-term supply of considerable financial resources, scientific and public-health vision, and dedication from all sectors of society. With these ingredients, the trajectory of our fight against the HIV/AIDS pandemic in the next quarter of a century could move from cautious optimism towards triumph. Absent any of these factors, and history will not judge us kindly.

Anthony S. Fauci is director of the National Institute of Allergy and Infectious Diseases (NIAID) at the US National Institutes of Health in Bethesda, Maryland, and chief of the NIAID Laboratory of Immunoregulation.

FURTHER READING

Barré-Sinoussi, F. et al. Science 220, 868-871 (1983).

Fauci, A. S. \& Lane, H. C. in Harrison's Principles of Internal

Medicine, 17 th edition (eds Fauci, A. S. et al.) 1137-1204

(McGraw Hill, New York, 2008).

Fauci, A. S. Science 239, 617-622 (1988).

Gallo, R. C. \& Montagnier, L. N. Engl. J. Med. 349, 2283-2285

(2003).

Joint United Nations Programme on HIV/AIDS (UNAIDS)

AIDS Epidemic Update: December 2007 (UNAIDS, Geneva,

2007).

Levy, J. A. HIV and the Pathogenesis of AIDS (ASM Press,

Washington DC, 2007). 